\title{
ESPECIES VEGETALES NATIVAS CON POTENCIAL PARA LA FITORREMEDIACIÓN DE SUELOS ALTO ANDINOS CONTAMINADOS POR RESIDUOS DE ACTIVIDAD MINERA
}

\author{
Luis Martínez-Manchego ${ }^{1}$, Guido Sarmiento-Sarmiento ${ }^{1}$ y Edwin Bocardo-Delgado ${ }^{1}$
}

\begin{abstract}
RESUMEN
La implementación de fitotecnologías para minimizar el impacto ambiental negativo de relaves mineros requiere caracterizar especies vegetales nativas adaptadas y tolerantes a suelos contaminados con metales tóxicos. El objetivo fue identificar y caracterizar especies vegetales nativas con potencial para la fitorremediación de suelos alto andinos contaminados por residuos de la actividad minera. La investigación se desarrolló en la zona de depósito de relaves de la empresa minera "El Madrigal" en el distrito de Madrigal, provincia Caylloma, Arequipa, Perú; ubicado a $15^{\circ} 35^{\prime}$ S, $71^{\circ} 50^{\prime}$ W y 3400 m de altitud. Se establecieron cinco zonas de muestreo (Primera, A, B, C y D). La concentración de contaminantes en las muestras de suelo con relaves se detectó mediante espectrometría de emisión atómica de plasma acoplado inductivamente; la recolección e identificación de especies vegetales se desarrolló mediante un muestreo sistemático estratificado y las muestras botánicas se enviaron al Instituto Científico Michael Owen Dillon para su identificación taxonómica según la propuesta del Grupo de Filogenia de Angiospermas. Para la caracterización de las especies vegetales se emplearon índices de similitud, diversidad y equitabilidad. Las especies vegetales más abundantes, y como tal, con mayor potencial de fitorremediación, fueron Cortaderia jubata, Baccharis sp., Stipa ichu y Juncus sp. También mostró alto potencial la especie Eragrostis nigricans, la cual fue identificada únicamente en la zona Primera, es decir, la zona con notable descarga de relaves mineros. En consecuencia, estas especies nativas mostraron mayor aptitud potencial para la fitorremediación de suelos contaminados con $\mathrm{As}, \mathrm{Ba}, \mathrm{Hg}, \mathrm{Cd}$ y $\mathrm{Pb}$ debido a su adaptación y tolerancia a los efectos adversos de la contaminación en estos suelos alto andinos.
\end{abstract}

Palabras clave adicionales: Fitotecnologías, plantas tolerantes, relaves mineros, remediación de suelos

\begin{abstract}
Native plant species with potential for phytoremediation of high-andean soils contaminated by residues from mining activity The implementation of phytotechnologies to minimize the negative environmental impact of mining tailings requires the characterization of native plant species adapted and tolerant to soils contaminated with toxic metals. The objective was to identify and characterize native plant species with potential for phytoremediation of high Andean soils contaminated by mining tailings. The research was carried out in the tailings deposit area of the mining company "El Madrigal" in the district of Madrigal, Caylloma province, Arequipa, Peru; located at $15^{\circ} 35^{\prime} \mathrm{S}, 71^{\circ} 50^{\prime} \mathrm{W}$ and $3400 \mathrm{~m}$ altitude. Five sampling zones were established (First, A, B, C and D). The concentration of contaminants in the soil samples with tailings was detected by inductively coupled plasma atomic emission spectrometry; the collection and identification of plant species was achieved by stratified systematic sampling, and the botanical samples were sent to the Michael Owen Dillon Scientific Institute for taxonomic identification according to the proposal of the Angiosperm Phylogeny Group. Similarity, diversity and equitability indices were used to characterize plant species. The most abundant plant species, and as such, with greater potential for phytoremediation were Cortaderia jubata, Baccharis sp, Stipa ichu and Juncus sp.; also, the species Eragrostis nigricans was included in this group because it was identified only in the first zone, the one with notable discharge of mining tailings. Consequently, these native species showed greater potential aptitude for phytoremediation of soils contaminated with $\mathrm{As}, \mathrm{Ba}, \mathrm{Hg}, \mathrm{Cd}$ and $\mathrm{Pb}$ due to their adaptation and tolerance to adverse effects of contamination in high Andean soils.
\end{abstract}

Additional keywords: Mining tailings, phytotechnologies, soil remediation, tolerant plants

\section{INTRODUCCIÓN}

La deficiente gestión ambiental de residuos procedentes de las minerías ocasiona impactos

Recibido: Febrero 16, 2021

${ }^{1}$ Universidad Nacional de San Agustín de Arequipa. Unidad de Posgrado de la Facultad de Ciencias Naturales y Formales. Arequipa, Perú. e-mail: lmartinezm@unsa.edu.pe; gsarmientos@ unsa.edu.pe (autor de correspondencia); ebocardo@unsa.edu.pe negativos en el entorno ambiental con riesgos para la salud humana y sus actividades de carácter socioeconómico (Cruzado et al., 2021); uno de los recursos ambientales más afectados es el suelo

Aceptado: Julio 9, 2021
ad de Posgrado de la Facuta 
logrando el deterioro de sus atributos físicos, químicos y biológicos que limita el establecimiento de la vegetación por el menoscabo de su calidad (Guerrero y Pineda, 2016); el impacto más evidente en el suelo es ocasionado por el vertido directo de relaves mineros que generalmente contienen elementos contaminantes que alteran sus funciones (Puga et al., 2006).

La explotación minera es una de las principales actividades de la economía peruana, varias empresas desarrollan sus operaciones en asentamientos ubicados en zonas alto andinas sobre los $3200 \mathrm{msnm}$ de altitud donde la minería a tajo abierto realiza una deficiente disposición de sus relaves con consecuencias ecológicas muy preocupantes (Chang et al., 2018). A pesar que muchas de estas empresas mineras dejaron de operar, los residuos mineros que abandonaron se convierten en pasivos ambientales que actualmente representan un problema en zonas alto andinas que requiere de la implementación de tecnologías para su remediación.

Las operaciones extractivas de la minera "El Madrigal" actualmente están paralizadas, abandonando sus residuos mineros (relaves). Los minerales explotados fueron la calcopirita, galena, blenda, cuarzo y pirita para extraer cobre, zinc, plomo, plata y oro, utilizando el sistema de boca mina o socavón.

Una de las tecnologías empleadas para la recuperación de suelos contaminados es la fitorremediación, la cual ofrece gran potencial para la remoción de una diversidad de compuestos contaminantes inorgánicos y orgánicos (Poma y Valderrama, 2014), y es de gran aceptación por la población inmersa en problemas de remediación de suelos por su eficiencia, rentabilidad y fácil aplicación (Ali et al., 2013).

La efectividad de un programa de fitorremediación depende de una apropiada identificación de especies vegetales, pues es obvio que no todas pueden emplearse para este propósito (Iqbal et al., 2008). Las especies que prosperan en zonas de depósitos de relaves mineros son de particular interés debido a que pueden tolerar condiciones adversas para su crecimiento y desarrollo superando limitaciones extremas para lograr su adaptación (Bech et al., 2016).

Varios estudios demostraron que las especies vegetales nativas que se desarrollan en sitios contaminados tienen alto potencial de fitorremediación (Shu et al., 2002; Yoon et al., 2006; Bech et al., 2016). En Perú, se evidenciaron 22 especies vegetales nativas pertenecientes a 12 familias de plantas con potencial de fitorremediación (Cruzado et al., 2021); en la región andina de Ancash en Perú, Chang et al. (2018) encontraron que Werneria nubigena, Pennisetum clandestinum, Medicago lupulina, Juncus bufonius, Calamagrostis recta y Achyrocline alata demostraron elevada tolerancia comportándose como plantas acumuladoras de $\mathrm{Cd}$, $\mathrm{Pb}, \mathrm{Ni}, \mathrm{Zn}$ y $\mathrm{Cu}$.

Por su parte, Santos et al. (2016) refieren que Cistus ladanifer es una especie excluidora de $\mathrm{Al}$, Ag, Ba, Bi, Sr y Sb. Fernández et al. (2017) indican que Coincya monensis es hiperacumulador de Zn. Ruiz et al. (2013) detectaron que Ricinus communis actúa como excluyente de $\mathrm{Cd}, \mathrm{Cu}$, $\mathrm{Mn}, \mathrm{Pb}$ y $\mathrm{Zn}$. Carrillo y González (2006) refieren que Polygonum aviculare responde como hiperacumulador de $\mathrm{Zn}$ y $\mathrm{Ag}$ en relaves ácidos de minas. Pernía et al. (2019) reportan a Sphagneticola trilobata como acumuladora de cadmio. Zine et al. (2021) indican que Atriplex semibaccata, Vicia sativa, Launaea arborescens, Peganum harmala y Asparagus horridus son especies nativas fitoestabilizadoras de residuos mineros fosfatados. En Qatar, se identificaron especies nativas de monocotiledóneas y dicotiledóneas eficaces en la recuperación de zonas contaminadas con relaves mineros (AlThani y Yasseen, 2019).

Identificar y caracterizar el establecimiento natural de especies vegetales nativas que prosperan sobre depósitos de relaves mineros es decisivo para restituir el equilibrio de un ecosistema para fomentar su estabilidad $\mathrm{y}$ resiliencia (Gajić et al., 2018). En el Perú, la zona alto andina del departamento de Arequipa soporta riesgos ambientales por el emplazamiento de relaves mineros que requieren con urgencia la implementación de planes de remediación ambiental sostenible; en este contexto, la presente investigación fue diseñada con el objetivo de identificar y caracterizar especies vegetales nativas con potencial para ser empleadas en la fitorremediación de suelos alto andinos contaminados por la actividad minera. 


\section{MATERIALES Y MÉTODOS}

Ámbito de la investigación. La investigación se desarrolló en la zona de depósito de relaves de la empresa minera "El Madrigal", abandonada por 30 años, ubicada a la margen derecha del río Colca en el distrito de Madrigal, provincia de Caylloma, departamento de Arequipa, Perú, a $15^{\circ} 35^{\prime} \mathrm{S}, 71^{\circ} 50^{\prime} \mathrm{W}$ y $3400 \mathrm{~m}$ de altitud. La temperatura media anual alcanza a $12,8{ }^{\circ} \mathrm{C}$, con una mínima de $-6,6{ }^{\circ} \mathrm{C}$ entre junio y julio, y máxima de 13,8 a $16,0{ }^{\circ} \mathrm{C}$ entre enero y marzo, característico de zonas alto andinas. Los relaves se ubicaron en las quebradas adyacentes a la zona de explotación con una superficie que abarca un perímetro de $644 \mathrm{~m}$ y un área total de $27.000 \mathrm{~m}^{2}$.

Análisis del terreno de investigación. Se identificaron seis puntos de muestreo para la toma de muestras de suelo con relave; en cada punto fue retirada una capa superficial de material a $30 \mathrm{~cm}$ de profundidad, las seis muestras fueron colocadas en bolsas de plástico para obtener una mezcla homogénea y mediante el método del cuarteo se obtuvo una muestra representativa de $1 \mathrm{~kg}$ para su envío a laboratorio (MINAM, 2014). Las determinaciones de elementos contaminantes en las muestras se realizaron en el Laboratorio de Ensayo y Control de Calidad de la Universidad Católica de Santa María de Arequipa, Perú, mediante el método de espectrometría de emisión atómica por plasma acoplado inductivamente (ICP-AES), marca OES Perkin-Elmer, mod. Optimo 8000.

Delimitación de la zona de muestreo. El área de investigación fue delimitada en cinco zonas: Primera o circunferencia, zona $\mathrm{A}$, zona $\mathrm{B}$, zona $\mathrm{C}$ y Zona D. La demarcación de la zona Primera se realizó proyectando una circunferencia desde la parte central del depósito de relaves con un radio de $21,8 \mathrm{~m}$; la zona A se delimitó al lado del depósito de relaves, y a continuación se delimitaron de forma contigua las zonas B, C y D, cada una con un área aproximada de $2400 \mathrm{~m}^{2}$.

Muestreo, recolección e identificación de especies vegetales. Se empleó un muestreo sistemático estratificado, el cual implicó la toma de muestras siguiendo un patrón sistemático al interior de cada estrato que permite detectar variaciones dentro de ellos; su aplicación en campo es sencilla y según el patrón espacial de los individuos ofrece mejor estimación que el muestreo sistemático sin estratificar (Sánchez, 1998).

Además, se consideró el muestreo selectivo y el método de la línea (Naoki et al., 2014) que consistió en escoger sitios con diferencias obvias o típicas; tales disparidades se determinaron mediante apreciación visual y criterios técnicos del evaluador (Halloy et al., 2011); se consideró como factores los cambios de color superficial del suelo, las áreas de perturbación física, áreas sin vegetación y con vegetación muerta. Se registró información referente a localidad, altitud, fecha de colección, hábitat, forma de vida o hábito, estado fenológico y datos morfológicos de importancia taxonómica. En cada zona de muestreo se contabilizó el número de especies; se recolectaron y prensaron especímenes para ser llevados al laboratorio para su posterior identificación; paralelamente se efectuó un registro fotográfico. Las muestras botánicas se enviaron al Instituto Científico Michael Owen Dillon (IMOD) para su identificación taxonómica según la propuesta del Grupo de Filogenia de Angiospermas (Chase et al., 2016).

Índice de similitud. Las semejanzas entre comunidades vegetales pueden ser expresadas numéricamente mediante el índice de Jaccard; este índice es comúnmente utilizado y se basa en la relación de presencia o ausencia entre el número de especies comunes en dos áreas respecto al número total de especies (Soler et al., 2012).

Índice de Jaccard: IJ $=\frac{c}{a+b-c}$, donde; a: es el número de especies en el sito $\mathrm{A}$; b: es el número de especies en el sito $\mathrm{B}$ y c: es el número de especies comunes en ambos sitios.

Índices de diversidad. Describen la diversidad de un determinado lugar, considerando el número de especies (riqueza) y el número de individuos de cada especie. El índice comúnmente utilizado es el de Simpson (Pla, 2006).

Índice de Simpson: $\mathrm{S}=1 / \sum\left(\frac{\mathrm{ni}(\mathrm{ni}-1)}{\mathrm{N}(\mathrm{N}-1)}\right)$

donde, ni: número de individuos en la especie y $\mathrm{N}$ : número total de individuos.

Índice de equitabilidad. Tiene como fundamento el concepto de equidad, uno de los más utilizados es el índice de Pielou el cual determina la proporción de la diversidad observada con respecto a la máxima diversidad esperada. $\mathrm{Su}$ valor va de 0 a 1 ; un valor de 1 corresponde a una condición en la cual todas las especies 
son igualmente abundantes (Mostacedo y Fredericksen, 2000).

Índice de Pielou: $\mathrm{J}=\frac{\mathrm{H}}{\log _{2} \mathrm{~S}}$

donde, $\mathrm{H}$ : índice de Shannon-Wiener y $\mathrm{S}$ : es el número máximo de especies en la muestra.

Tratamiento estadístico. Para la determinación de los índices de similitud Jaccard, los índices de diversidad de Shannon-Wiener y Simpson y el índice de Pielou; se utilizó el programa estadístico PAST (Paleontological Statistic) versión 3.18.

\section{RESULTADOS Y DISCUSIÓN}

Identificación de elementos contaminantes en la zona de investigación. En el Cuadro 1 se muestran 22 elementos detectados en la composición de los relaves, de los cuales 10 (P, K, $\mathrm{Mg}, \mathrm{Ca}, \mathrm{Mn}, \mathrm{B}, \mathrm{Cu}, \mathrm{Fe}, \mathrm{Zn}, \mathrm{Ni}$ ) son nutrientes esenciales empleados por diversas especies vegetales, mientras que el $\mathrm{Na}$ y $\mathrm{Si}$ son elementos benéficos solo para algunas plantas (Mengel y Kirkby, 2000; Alcántar y Trejo, 2010), siendo útiles para la nutrición mineral de las plantas identificadas en la zona de investigación. Por su parte, el aluminio está asociado a suelos ácidos, y en nuestra investigación, la flora nativa identificada toleró los niveles de este elemento; estos mecanismos de tolerancia de las plantas están relacionados al incremento de $\mathrm{pH}$ de la rizósfera y la eliminación del Al y ácidos orgánicos absorbidos (Yoshimura et al., 2003).

El litio es absorbido por algunas plantas estimulando el crecimiento, aunque no es esencial para su desarrollo; la absorción y tolerancia al litio dependen de la especie; generalmente las plantas absorben más litio en los suelos ácidos (Aral y Vecchio, 2008). Según Galán y Romero (2008), el As, $\mathrm{Ba}, \mathrm{Hg}, \mathrm{Cd}, \mathrm{Pb}, \mathrm{Sb}$ y Ti se consideran como elementos muy tóxicos y fácilmente disponibles en suelos en concentraciones que exceden los valores de toxicidad.

El vanadio participa en el crecimiento de algunas plantas cumpliendo funciones biológicas relacionadas al incremento de aminoácidos, azúcares y clorofila (Rojek et al., 2019); sin embargo, su exceso en el suelo ocasiona clorosis y retrasos en el crecimiento (Hou et al., 2019).

Según la normativa peruana (MINAM, 2017), los niveles de $\mathrm{As}$, $\mathrm{Ba}$ y $\mathrm{Hg}$ detectados en la presente investigación superan los estándares de calidad ambiental (ECA) para suelos dedicados a la actividad extractiva como la minería. Estos estándares son 140, 2000 y $24 \mathrm{mg} \cdot \mathrm{kg}^{-1}$ de As, Ba y $\mathrm{Hg}$, respectivamente, por lo que las especies vegetales identificadas fueron tolerantes a dichos elementos y podrían emplearse para la fitorremediación de estos suelos. En el caso de cadmio los niveles encontrados en la zona de investigación $\left(21,43 \mathrm{mg} \cdot \mathrm{kg}^{-1}\right)$ están cerca del ECA nacional $\left(22 \mathrm{mg} \cdot \mathrm{kg}^{-1}\right)$ por lo tanto, las plantas nativas identificadas tienen potencial para su uso en fitorremediación de suelos con este metal pesado.

Cuadro 1. Elementos detectados en la zona de depósito de relaves de la empresa minera "El Madrigal" provincia de Caylloma, departamento de Arequipa, Perú

\begin{tabular}{lr}
\hline $\begin{array}{r}\text { Elemento } \\
\text { detectado }\end{array}$ & $\begin{array}{r}\text { Concentración } \\
\left(\mathrm{mg}^{-1} \mathrm{~kg}^{-1}\right)\end{array}$ \\
\hline Silicio & $1.299 .071,43$ \\
Sodio & $52.864,29$ \\
Aluminio & $27.885,71$ \\
Hierro & $22.550,00$ \\
Calcio & $21.635,71$ \\
Potasio & $19.421,43$ \\
Boro & $17.892,86$ \\
Bario & $15.892,86$ \\
Zinc & $3.485,71$ \\
Magnesio & 885,71 \\
Plomo & 628,57 \\
Titanio & 371,43 \\
Fósforo & 335,71 \\
Arsénico & 307,14 \\
Manganeso & 142,86 \\
Vanadio & 50,00 \\
Cobre & 50,00 \\
Mercurio & 42,86 \\
Litio & 35,71 \\
Antimonio & 35,71 \\
Cadmio & 21,43 \\
Níquel & 14,29 \\
\hline
\end{tabular}

Caracterización de especies vegetales nativas en la zona de investigación. Los resultados expuestos en el Cuadro 2 refieren que en la investigación se identificaron 16 especies vegetales, agrupadas en siete familias (Asteraceae, Boraginaceae, Brasicaceae, Fabaceae, Juncaceae, Poaceae y Scrophulariacae) pertenecientes a cinco órdenes (Asterales, Boraginales, Brassicales, 
Fabales y Poales). La zona D presentó el mayor número de especies (12) y la zona $\mathrm{C}$ registró el mayor número total de individuos de todas las especies (221).

La tendencia de los resultados demuestra que en las distintas zonas de muestreo la vegetación nativa fue muy escasa, y el desarrollo de una mayor diversidad y mayor número de individuos se presentó conforme las zonas se alejan del punto medio del depósito de relave, desde la zona Primera hasta la zona C (Cuadro 2). En el caso de la zona $\mathrm{D}$ se observó una disminución del número de especies, situación que se justifica debido a que se encuentra bajo la influencia de factores antropogénicos, identificándose en el lugar un camino de acceso para trabajadores hacia el campamento de la mina.

En el Cuadro 2 se indica la composición de las especies vegetales identificadas, así como el número total de especies e individuos y el porcentaje de individuos en las cinco zonas de muestreo. En la zona Primera se identificaron las especies Cortaderia jubata, Eragrostis nigricans y Stipa ichu las cuales muestran especial importancia debido a que esta zona de muestreo fue el punto medular de descarga de relaves de la empresa minera. Baccharis sp. fue reconocida en cuatro de las zonas de muestreo.

Cuadro 2. Identificación de especies vegetales, número total de especies e individuos y porcentaje de individuos en las cinco zonas de muestreo

\begin{tabular}{|c|c|c|c|c|c|c|c|c|}
\hline \multirow{2}{*}{ Orden } & \multirow{2}{*}{ Familia } & \multirow{2}{*}{ Especie } & \multicolumn{5}{|c|}{ Zona } & \multirow{2}{*}{ Total } \\
\hline & & & Primera & A & B & $\mathrm{C}$ & $\mathrm{D}$ & \\
\hline Asterales & Asteraceae & Achyrocline alata (Kunth) DC. & - & - & - & - & 1 & 1 \\
\hline Asterales & Asteraceae & Baccharis sp. & - & 55 & 5 & 29 & 3 & 92 \\
\hline Asterales & Asteraceae & Grindelia tarapacana Phil. & - & - & - & - & 5 & 5 \\
\hline Asterales & Asteraceae & $\begin{array}{l}\text { Pseudognaphalium dombeyanum } \\
\text { (DC) Anderb. }\end{array}$ & - & - & - & - & 1 & 1 \\
\hline Boraginales & Boraginaceae & Phacelia secunda J.F. Gmel. & - & - & - & 5 & - & 5 \\
\hline Brassicales & Brasicaceae & $\begin{array}{l}\text { Descurainia myriophylla } \\
\text { (Willd. Ex DC) R.E.Fr. }\end{array}$ & - & - & - & - & 1 & 1 \\
\hline Fabales & Fabaceae & Cytisus sp. & - & - & - & - & 3 & 3 \\
\hline Fabales & Fabaceae & Lupinus paruroensis C.P.Sm. & - & 10 & - & - & - & 10 \\
\hline Fabales & Fabaceae & Spartium junceum L. & - & - & - & - & 2 & 2 \\
\hline Poales & Juncaceae & Juncus sp. & - & - & - & 30 & 20 & 50 \\
\hline Poales & Poaceae & Cortaderia jubata (Lemoine) Stapf. & 27 & 11 & 152 & 104 & 66 & 360 \\
\hline Poales & Poaceae & Eragrostis nigricans (Kunth) Steud. & 14 & - & - & - & - & 14 \\
\hline Poales & Poaceae & Eragrostis sp. & - & - & - & - & 2 & 2 \\
\hline Poales & Poaceae & $\begin{array}{l}\text { Kikuyuochloa clandestina (Hochst. } \\
\text { Ex Chiov) H. Scholz. }\end{array}$ & - & - & & 30 & - & 30 \\
\hline Poales & Poaceae & Stipa ichu (Ruiz \&Pav) Kunth & 3 & 21 & 8 & 23 & 7 & 62 \\
\hline Poales & Scrophulariacae & Buddleja coriácea J. Rémy & - & - & - & - & 1 & 1 \\
\hline \multicolumn{3}{|c|}{ Total de especies } & 3 & 4 & 3 & 6 & 12 & - \\
\hline \multicolumn{3}{|c|}{ Total de individuos } & 44 & 97 & 165 & 221 & 112 & 639 \\
\hline \multicolumn{3}{|c|}{ Porcentaje de individuos } & 6,9 & 15,2 & 25,8 & 34,6 & 17,5 & - \\
\hline
\end{tabular}

Las especies exclusivas por cada zona de muestreo fueron una especie en la zona Primera (E. nigricans), uno en la zona A (L. paruroensis), dos especies en la zona C ( $P$. secunda y $K$. clandestina) y ocho en la zona D. La zona B no registró especies exclusivas. De acuerdo al número total de individuos encontrados en las diferentes zonas (Cuadro 2), las especies más abundantes, y como tal, con potencial de fitorremediación fueron C. jubata, Baccharis sp., S. ichu y Juncus sp. A este grupo hay que agregar la especie E. nigricans, la cual, a pesar de no 
presentar una abundancia alta, tuvo todos su individuos en la zona Primera, es decir, la zona con notable descarga de relaves mineros.

Según la Figura 1, la especie $C$. jubata representa el mayor porcentaje $(56,34 \%)$ del total de individuos identificados por cada especie; en cambio, A. alata, P. dombeyanum, D. myriophylla y B. coriacea son especies con menor presencia, cada una con solo $0,16 \%$ de representatividad del total. Las actividades humanas, como la explotación minera mal gestionada, alteran la biodiversidad y estabilidad de ecosistemas debido a la disminución de especies (Hautier et al., 2015). Algunas especies identificadas fueron reportadas en investigaciones realizadas en terrenos de los
Andes centrales del Perú, donde de manera directa o indirecta la actividad minera influye en el entorno mediante sus pasivos ambientales (Chang et al., 2018). Yarupaitán y Albán (2003) identificaron las familias Asteraceae y Poaceae y el género Baccharis sp. como la flora más importante en los Andes centrales de Junín; así mismo, Yaranga et al. (2018) en un estudio realizado en los andes de Junín reportaron $S$. ichu y Baccharis sp. que corresponden a las familias Poaceae y Asteraceae respectivamente. Por su parte, Gutiérrez y Castañeda (2014) reportaron Baccharis sp. y E. nigricans en una investigación desarrollada en zona alto andina de Huancavelica en Perú.

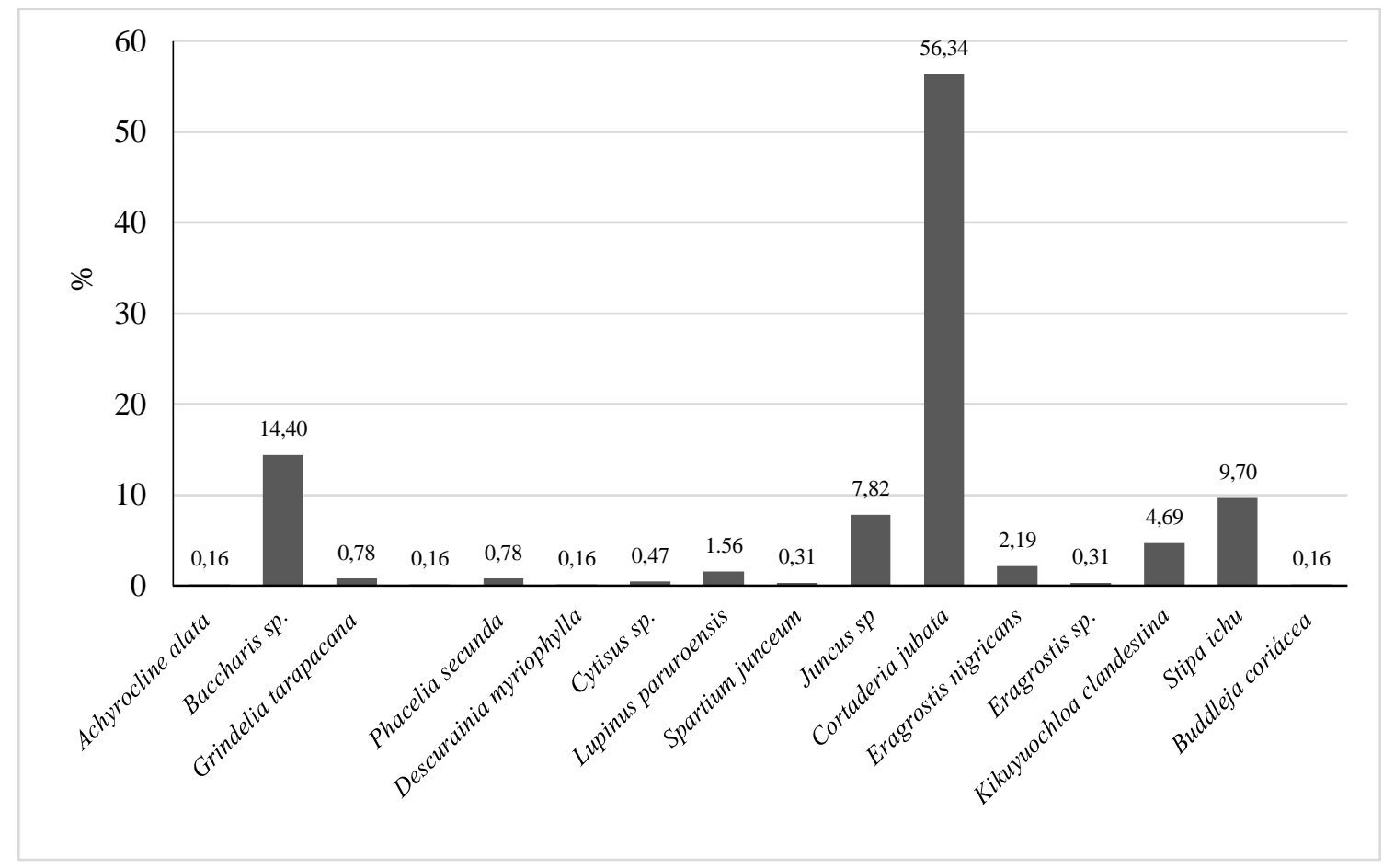

Figura 1. Composición porcentual de individuos por cada especie con potencial para fitorremediación identificada en la zona de estudio

La identificación de especies vegetales presentada en el Cuadro 2 se complementa con un análisis técnico de la diversidad florística encontrada en la zona de estudio en base a los índices de similitud, índice de diversidad y el índice de equitabilidad que resultan muy convenientes frente a una transformación dinámica de los ecosistemas naturales (Wiesmair et al., 2017).

El Cuadro 3 ofrece la similitud de la presencia de especies según el índice de Jaccard. Se aprecia que las comparaciones entre la zona Primera y la zona $\mathrm{B}$, y entre ésta y la zona $\mathrm{C}$ presentan un índice de 0,5; y entre zonas A y B un índice de 0,75; estos índices determinan una semejanza media y elevada de las especies identificadas entre estas zonas, y existen varias especies compartidas entre ellos; las demás interacciones o comparaciones entre zonas evaluadas presentan índices bajos por lo que la presencia común de algunas especies entre ellos no es muy apreciable, 
demostrando heterogeneidad de especies entre estas zonas.

La baja similaridad florística entre las zonas de muestreo evaluadas puede atribuirse a las condiciones edáficas peculiares de cada zona; en la investigación, la zona Primera y zona A incluyen suelos con descarga de manera directa del relave de la empresa minera.

Cuadro 3. Matriz de Presencia/Ausencia de las especies vegetales encontradas en la zona de investigación considerando el índice de Jaccard

\begin{tabular}{lccccc}
\hline \multicolumn{1}{c}{ Zona } & Primera & A & B & C & D \\
\hline Primera & 1 & - & - & - & - \\
A & 0,40 & 1 & - & - & - \\
B & 0,50 & 0,75 & 1 & - & - \\
C & 0,29 & 0,43 & 0,50 & 1 & - \\
D & 0,15 & 0,23 & 0,25 & 0,29 & 1 \\
\hline
\end{tabular}

La Figura 2 revela el dendrograma de similitud construido a partir del índice de Jaccard $(\mathrm{R}=0,9485)$. Se aprecia que existen tres grupos establecidos en la zona de estudio; uno conformado por las zonas Primera, A y B que presentan el menor número de especies por estar ubicados en las cercanías del punto medio de descarga del deslave. Los otros dos grupos conformados, por las zonas $\mathrm{C}$ y $\mathrm{D}$, fueron presentando un mayor número de especies en la medida que se alejeaban del foco de contaminación.

Los resultados del índice de diversidad o dominancia de Simpson (Cuadro 4) indican que la zona $\mathrm{C}$ obtuvo el mayor índice $(0,7131)$, por lo que esta zona presenta mayor riqueza de especies dominantes y es más homogénea respecto a las otras zonas de muestreo; en cambio, la zona B mostró el menor índice $(0,1481)$, lo que evidencia baja uniformidad en la distribución de individuos entre las especies dominantes de esta zona.



Figura 2. Dendrograma de similitud construido a partir del índice de Jaccard para la presencia de especies en la zona de estudio
El índice de equitabilidad de Pielou (Cuadro 4) muestra el mayor valor para la zona $\mathrm{C}$ $(0,8286)$ indicando que el $82,86 \%$ de especies identificadas en esta zona son igualmente abundantes; en cambio, en la zona B solo el $29,88 \%$ de especies son igualmente abundantes. Valores cercanos a cero indican que una especie domina sobre todas las demás, mientras que si es cercano a 1 corresponde a situaciones donde todas las especies son igualmente abundantes (Soler et al., 2012).

Cuadro 4. Índices de Simpson y Pielou correspondientes a las especies encontradas en la zona de estudio

\begin{tabular}{lccccc}
\hline \multirow{2}{*}{ Índices } & \multicolumn{4}{c}{ Zona } \\
\cline { 2 - 6 } & Primera & $\mathrm{A}$ & $\mathrm{B}$ & $\mathrm{C}$ & $\mathrm{D}$ \\
\hline Simpson & 0,5176 & 0,6081 & 0,1481 & 0,7131 & 0,6121 \\
Pielou & 0,7711 & 0,8181 & 0,2988 & 0,8286 & 0,6115 \\
\hline
\end{tabular}

\section{CONCLUSIONES}

Las especies vegetales más abundantes de la zona de investigación fueron Cortaderia jubata, Baccharis sp., Stipa ichu y Juncus sp., junto a la especie Eragrostis nigricans que fue identificada únicamente en la zona Primera, donde existe la mayor descarga de relaves minero. Esto indica que todas estas especies nativas, al tolerar los efectos adversos de la contaminación, ofrecen mayor aptitud potencial para su utilización en la fitorremediación de suelos contaminados esencialmente con As, $\mathrm{Ba}, \mathrm{Hg}, \mathrm{Cd}$ y $\mathrm{Pb}$.

Se deben continuar con investigaciones a fin de determinar la capacidad de remoción de 
contaminantes por cada especie vegetal identificada. Esta información puede aportar al desarrollo e innovación de fitotecnologías para gestionar la remediación de suelos contaminados en zonas altoandinas.

\section{LITERATURA CITADA}

1. Alcántar, G. y L. Trejo-Téllez. 2010. Nutrición de Cultivos. Mundi-Prensa. México.

2. Ali, H., E. Khan y M. Sajad. 2013. Phytoremediation of heavy metals.Concepts and applications. Chemosphere 91(7): 869881.

3. Al-Thani, R.F. y B.T. Yasseen. 2019. Phytoremediation of polluted soils and waters by native Qatari plants: Future perspectives. Environmental Pollution 259: 113694.

4. Aral, H. y A. Vecchio-Sadus. 2008. Toxicity of lithium to humans and the environment: A literature review. Ecotoxicology and Environmental Safety 70(1): 349-356.

5. Bech, J., N. Roca, P. Tume, J. Ramos-Miras, C. Gil y R. Boluda. 2016. Screening for new accumulator plants in potential hazards elements polluted soil surrounding Peruvian mine tailings. Catena 136: 66-73.

6. Carrillo-González, R. y M. González-Chávez. 2006. Metal accumulation in wild plants surrounding mining wastes. Environmental Pollution 144(1): 84-92.

7. Chang, J., M. Gonzales, O. Ponce, L. Ramírez, V. León, A. Torres, M. Corpus y R. Loayza-Muro. 2018. Acumulación de metales pesados en plantas nativas andinas: herramientas potenciales para la fitorremediación de suelos en Ancash (Perú). Environmental Science and Pollution Research 25(34): 33957-33966.

8. Chase, M.W., M.J. Christenhusz, M.F. Fay, J.W. Byng, W.S. Judd, D.E. Soltis et al. 2016. The Angiosperm Phylogeny Group: An update of the Angiosperm Phylogeny Group classification for the orders and families of flowering plants - APG IV. Botanical Journal of the Linnean Society 181(1): 1-20.

9. Cruzado-Tafur, E., L. Torró, K. Bierla, J. Szpunar y E. Tauler. 2021. Contenido de metales pesados en suelos e inventario de flora nativa en pasivos ambientales mineros en los Andes peruanos. Revista de Ciencias de la Tierra de América del Sur 106: 103-107.

10.Fernández, S., C. Poschenrieder, C. Marceno, J. Gallego, D. Jiménez-Gámez, A. Bueno y E. Afif. 2017. Phytoremediation capability of native plant species living on $\mathrm{Pb}-\mathrm{Zn}$ and $\mathrm{Hg}$-As mining wastes in the Cantabrian range, north of Spain. Journal of Geochemical Exploration 174: 10-20.

11.Gajić, G., L. Djurdjević, O. Kostić, S. Jarić, M. Mitrović y P. Pavlović. 2018. Ecological potential of plants for phytoremediation and ecorestoration of fly ash deposits and mine waste. Frontiers in Environmental Science 6: 124.

12.Galán, E. y A. Romero. 2008. Contaminación de suelos por metales pesados. Macla: Revista de la Sociedad Española de Mineralogía 10(1): 48-60.

13. Guerrero, M. y V. Pineda. 2016. Contaminación del suelo en la zona minera de Rasgatá Bajo (Tausa), modelo conceptual. Ciencia e Ingeniería Neogranadina 26(1): 57-74.

14.Gutiérrez, H. y R. Castañeda. 2014. Diversidad de las gramíneas (Poaceae) de Lircay (Angaraes, Huancavelica, Perú). Ecología Aplicada 13(1): 23-33.

15.Halloy, S., M. Ibáñez y K. Yager. 2011. Puntos y áreas flexibles (PAF) para inventarios rápidos del estado de biodiversidad. Ecología en Bolivia 46(1): 46-56.

16.Hautier, Y., D. Tilman, F. Isbell, E. Seabloom, E. Borer y P. Reich. 2015. Anthropogenic environmental changes affect ecosystem stability via biodiversity. Science 348(6232): 336-340.

17.Hou, M., M. Li, X. Yang y R. Pan. 2019. Responses of nonprotein thiols to stress of vanadium and mercury in maize (Zea mays L.). Bulletin of Environmental Contamination and Toxicology 102: 425-431.

18.Iqbal, M., Z. He, P. Stoffella y X. Yang. 2008. Phytoremediation of heavy metal polluted soils and water: Progresses and perspectives. Journal of Zhejiang University Science 9(1): 210-220. 
19.Mengel, E. y E. Kirkby. 2000. Principios de Nutrición Vegetal. Instituto Internacional del Potasio. Suiza.

20.MINAM (Ministerio del ambiente- Perú). 2014. Guía para el muestreo de suelos. https://n9.cl/4yfyz (consulta de Dic. 4, 2020).

21.MINAM (Ministerio del Ambiente, Perú). 2017. Estándares de calidad ambiental para suelos - ECA. Decreto Supremo N ${ }^{\circ} 011-2017-$ MINAM. https://n9.cl/u6omg (consulta de Octubre 4, 2020).

22. Mostacedo, B. y T. Fredericksen. 2000. Manual de métodos básicos de muestreo y análisis en ecología vegetal. El País. La Paz. $87 \mathrm{p}$.

23.Naoki, K., R. Meneses, M. Gómez y C. Landivar. 2014. El uso del método de puntos de intercepción para cuantificar los tipos de vegetación y hábitats abióticos en los bofedales altoandinos. Ecología en Bolivia 49(3): 84-90.

24.Pernía, B., M. Calabokis, K. Noris, J. Bubis, M. Guerra y M. Castrillo. 2019. Effects of cadmium in plants of Sphagneticola trilobata (L.) Pruski. Bioagro 31(2): 133-142.

25.Pla, L. 2006. Biodiversidad: Inferencia basada en el índice de Shannon y la riqueza. Interciencia 31(8): 583-590.

26.Poma, V. y Valderrama, A. 2014. Estudio de los parámetros fisicoquímicos para la fitorremediación de cadmio (ii) y mercurio (ii) con la especie Eichhornia crassipes (jacinto de agua). Revista de la Sociedad Química del Perú 80(3): $164-173$.

27.Puga, S., M. Sosa, T. Lebgue, C. Quintana y A. Campos. 2006. Contaminación por metales pesados en suelo provocada por la industria minera. Ecología Aplicada 5(1-2): 149-155.

28.Rojek, J., M. Kozieradzka-Kiszkurno, M. Kapusta, A. Aksmann, D. Jacewicz, J. Dzon et al. 2019. The effect of vanadium (IV) complexes on development of Arabidopsis thaliana subjected to $\mathrm{H}_{2} \mathrm{O}_{2}$-induced stress. Funct. Plant. Biol. 46(10): 942-961.

29.Ruiz-Olivares, A., R. Carrillo-Gonzales, R.M. Gonzales-Chávez y R. Soto. 2013. Potencial de ricino (Ricinus communis L.) para la fitorremediación de relaves de minas y producción de aceite. J. Environmental Management 114: 316 -323.

30.Sánchez-Crespo, G. 1998. Muestreo sistemático con intervalo de selección variable. Estadística Española 40(143): 5-31.

31.Santos, E., M. Abreu y M. Magalhaes. 2016. Cistus ladanifer phytostabilizing soils contaminated with non-essential chemical elements. Ecological Engineering 94: 107-116.

32.Shu, W., Z. Ye, C. Lan, Z. Zhang y M. Wong. 2002. Lead, zinc and copper accumulation and tolerance in populations of Paspalum distichum and Cynodon dactylon. Environ. Pollution 120: 445- 453.

33. Soler, P., J. Berroterán, J. Gil y R. Acosta. 2012. Índice valor de importancia, diversidad y similaridad florística de especies leñosas en tres ecosistemas de los llanos centrales de Venezuela. Agronomía Tropical 62(1-4): 2538.

34.Wiesmair, M., A. Otte y R. Waldhardt. 2017. Relationships between plant diversity, vegetation cover, and site conditions: implications for grassland conservation in the Greater Caucasus. Biodiversity and Conservation 26(2): 273-291.

35. Yaranga, R., M. Custodio, F. Chanamé y R. Pantoja. 2018. Diversidad florística de pastizales según formación vegetal en la subcuenca del río Shullcas, Junín, Perú. Scientia Agropecuaria 9(4): 511- 517.

36. Yarupaitán, G. y J. Albán. 2003. Flora silvestre de los Andes centrales del Perú: un estudio en la zona de Quilcas, Junín. Revista Peruana de Biología 10(2): 155-162.

37.Yoon, J., X. Cao, Q. Zhou y L. Ma. 2006. Accumulation of $\mathrm{Pb}, \mathrm{Cu}$, and $\mathrm{Zn}$ in native plants growing on a contaminated Florida site. Science of the Total Environment 368(2-3): 456-464.

38. Yoshimura, E., T. Nakagava y S. Mori. 2003. Distribution and chemical forms of aluminumtolerant rice cultivar grown with or without phosfate pretreatment. Comunications in Soil Science and Plant Analysis 34(11-12): 15491555. 
39. Zine, H., S. Elgadi, R. Hakkou, E. phosphate mine waste in semi-arid Papazoglou, L. Midhat y A. Ouhammou. 2021. environments: a field experiment. Minerals Wild plants for the phytostabilization of 11(42): 1-15. 